\title{
High Sensitive Chiral Molecule Detector Based on The Amplified Lateral Shift in Kretschmann Configuration Involving Chiral TDBCs
}

\section{Song Wang}

Beijing University of Posts and Telecommunications

Qihui Ye

Beijing University of Posts and Telecommunications

\section{Xudong Chen}

Beijing University of Posts and Telecommunications

\section{Yanzhu Hu}

Beijing University of Posts and Telecommunications

\section{Gang Song ( $\nabla$ sg2010@bupt.edu.cn )}

Beijing University of Posts and Telecommunications https://orcid.org/0000-0001-8774-9337

\section{Nano Express}

Keywords: Surface plasmon polaritons, Goos-Hanchen shift, Chirality

Posted Date: December 9th, 2020

DOl: https://doi.org/10.21203/rs.3.rs-122144/v1

License: (c) (1) This work is licensed under a Creative Commons Attribution 4.0 International License. Read Full License

Version of Record: A version of this preprint was published at Chinese Physics B on June 1st, 2021. See the published version at https://doi.org/10.1088/1674-1056/abdda6. 


\title{
High sensitive chiral molecule detector based on the amplified lateral shift in Kretschmann configuration involving chiral TDBCs
}

\author{
Song Wang, Qihui Ye, Xudong Chen, \\ Yanzhu Hu • Gang Song
}

Received: date / Accepted: date

\begin{abstract}
We investigate a high sensitive chiral molecule detector based on Goos-Hanchen shift $(S)$ in Kretschmann configuration involving chiral TDBCs. Fresnel equations and the stationary phase method are employed to calculate $S$. Due to the interaction between surface plasmon polaritons and chiral TDBCs, $S$ with chiral TDBCs are amplified at near the resonant wavelengths of chiral TDBCs. Our calculation results show that although the difference between the resonant wavelengths of left and right TDBCs is $4.5 \mathrm{~nm}$, the difference of $S$ with chiral TDBCs $(\Delta S)$ can reach to 400 times as the incident wavelength in certain conditions, which can be easily observed in experiments. There is an optimal thickness of the metal film to realize the largest difference of $S$ between Kretschmann configurations with left TDBCs and right TDBCs. We also find that the positions of the largest $S$ for the structures with left TDBCs and right TDBCs do not overlap. Furthermore, we discuss the oscillator strength $f$, which is mainly determined by TDBC concentration. We find that our proposed detector is quite sensitive with $f$. By changing $f$ from 0.008 to 0.014 with the step of 0.002 , the change of $\Delta S$ is no less than 5 times of the incident wavelength $(2.9 \mu \mathrm{m})$. Our proposed structure is very sensitive and has potential applications in experiments.
\end{abstract}

Keywords Surface plasmon polaritons · Goos-Hanchen shift · Chirality

S. Wang, Y. Z. Hu

School of Modern Post, BUPT, Beijing, China

E-mail: wongsang@bupt.edu.cn

X. D. Chen, Q. H. Ye, G. Song

School of Science, Beijing University of Posts and Telecommunications (BUPT), Beijing 100876, China

E-mail: sg2010@bupt.edu.cn

Q. H. Ye

International School, BUPT, Beijing, China 


\section{Introduction}

During the past couples of years, the optical sensors at macro- and nano- scales have been investigate, and surface plasmon polaritons (SPPs) play an important role in these devices [1-12]. Goos-Hänchen (GH) shift is a small shift of light in the total internal reflection which is quite sensitive to the surrounding medium [1-10,12]. Many applications are introduced in both theory and experiments such as sensors [3-5], resonance devices [6] and switches based on Kretschmann configuration involving Kerr nonlinear medium [7]. Kretschmann configuration is a well known configuration in SPP excitation [7,13-16,12]. GH shift can directly measure in experiment and can reach to 100 times as the incident wavelength [7]. If the medium in Kretschmann configuration is chiral, GH would display a new function for detecting the chirality of the medium.

A chiral object does not have mirror planes or inversion symmetry [17]. Each of such non-superimposable mirror images is known as an enantiomer, and can be divided into left- and right-handedness, respectively. Chiral objects generally display similar physical properties to distinguish them[18]. SPPs and localized surface plasmons (LSPs) can enhance the light field in the near field [19-39]. With the help of the enhancement of electric field, the chiral optical response can be significantly amplified in chiral systems in form of the circular dichroism (CD) spectra enhancement [19-33]. If we use GH shift to replace the optical response such as the absorption and the extinction, the difference between the left molecules and the right molecules can be easily measured in experiment.

In this paper, we investigate a chiral molecule detector based on the lateral shift splitting/GH shift splitting in Kretschmann configuration involving chiral TDBCs by using classical methods. Kretschmann configuration is made up of a gold film sandwiched between the dielectric medium and left TDBCs or right TDBCs. Incident light is injected from the dielectric medium with an incident angle. Fresnel equations and the stationary phase method are employed to solve our proposed structure. The parameters of chiral TDBCs and Au film are changed in order to show the impact on the sensitivity of the molecule detector.

\section{Calculation Model and Analysis}

The sketch of our calculation model is shown in Fig. 1. An Au film with the thickness of $d$ is sandwiched between the prism and chiral TDBCs. Incident chiral beam is injected from the dielectric medium with an incident angle $\theta$. The dielectric constant function $\left(\epsilon_{2}\right)$ of $\mathrm{Au}$ is obtained in the Ref. [40]. The index of the dielectric medium $\left(\sqrt{\epsilon_{1}}\right)$ is chose as $\sqrt{3.6}$. Chiral TDBCs are modeled by a Lorentzian oscillator $[29,30]$ :

$$
\epsilon_{3}=\epsilon_{\infty}+\frac{f_{i} \omega_{i}^{2}}{\omega_{i}^{2}-\omega^{2}-i \gamma_{i} \omega}
$$




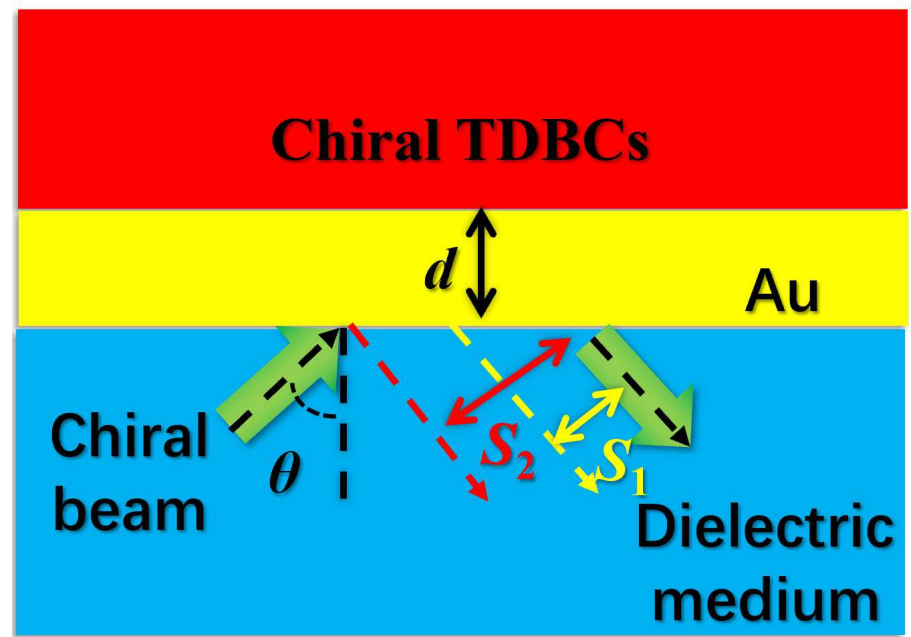

Fig. 1 (Color online). The sketch of our calculation model.

where, $i$ means Left (L) or Right (R). $\epsilon_{\infty}=n_{b g}^{2}$, and $n_{b g}=1.33$ is the index of the background. $\omega_{i}$ is the resonant frequency of of TDBCs, and $\gamma_{i}$ is the damping constant of TDBC. $f_{i}$ is the oscillator strength, which depends on the molecular concentration. According to the experiment measuremen$\mathrm{t}$ of TDBC, we obtain that $\omega_{L}=3.228 \times 10^{15}[\mathrm{rad} / \mathrm{s}], \omega_{R}=3.203 \times 10^{15}[\mathrm{rad} / \mathrm{s}]$, and $\gamma_{L}=\omega_{R}=8.195 \times 10^{13}[\mathrm{rad} / \mathrm{s}][29,30]$. Incident chiral beam is composed of TE-polarized beam $\left(\mathbf{E}_{s}\right)$ and TM-polarized beam $\left(\mathbf{E}_{p}\right)$ as $\mathbf{E}_{s}+\mathbf{E}_{p} e^{i \pm \pi / 2}$. In Kretschmann configurations, only TM-polarized beam can be used to generate SPPs. Here, we only consider TM-polarized beam is injected in our proposed system.

GH shift $S$ is calculated by the classical method. According to the Fresnel equations of this kind of Kretschmann configurations, the reflection coefficients of TM-polarized are written as [7,12]:

$$
r=\frac{r_{12}+r_{23} \exp \left(2 i k_{2} d\right)}{1+r_{12} r_{23} \exp \left(2 i k_{2} d\right)}
$$

where the order of the layers 1, 2 and 3, counted from bottom to top and

$$
r_{a b}=\frac{\epsilon_{b} k_{a}-\epsilon_{a} k_{b}}{\epsilon_{b} k_{a}+\epsilon_{a} k_{b}}, a, b=1,2,3
$$

and, $k_{a}=\sqrt{k_{0}^{2} \epsilon_{a}-k_{\perp}^{2}}, k_{\perp}=k_{0} \sqrt{\epsilon_{1}} \sin \theta$ and $k_{0}$ is the vacuum wave vector. The reflection $R$ is calculated as $R=|r|^{2}$. According to the stationary phase method, the lateral shift of the reflected beam $S$ is given by [2,7]:

$$
S=\frac{1}{\sqrt{\epsilon_{1}} k_{0}}\left|\frac{d \phi_{r}}{d \theta}\right|
$$



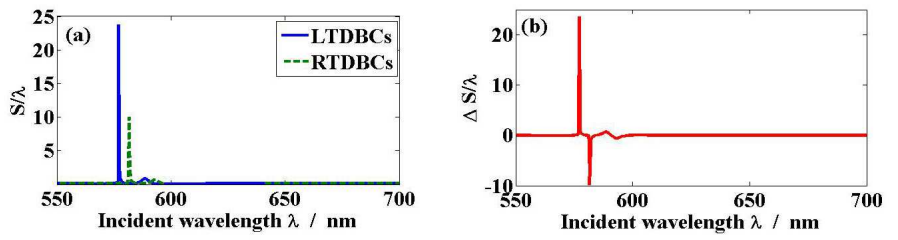

Fig. 2 Normalized GH shifts $S_{i} / \lambda$ (a) and the difference of normalized GH shift $\Delta S / \lambda(\mathrm{b})$ versus the incident wavelength $\lambda$, respectively.

where, $\phi_{r}=\Im(\ln r)$ is the phase of $r$. Before we discuss the relation between the GH shift $S$ and the incident wavelength $\lambda$, it should be mentioned that the incident angle $\theta$ is equal to the surface plasmon resonant angle $\theta_{S P P s}[2$, 7]. Here, we adopt $\theta_{S P P s}$ as the incident angle $\theta$ for each incident wavelength $\lambda$ written as [41]:

$$
\sin \theta=\sin \theta_{S P P s}=\left|\sqrt{\frac{\epsilon_{2} \epsilon_{3}}{\epsilon_{2}+\epsilon_{3}}} \epsilon_{1}^{-\frac{1}{2}}\right| .
$$

In the following, we calculate Kretschmann configurations with only LTDBCs and Right TDBCs to do the research on normalized GH shift $S / \lambda$ and the difference of normalized GH shifts between Kretschmann configurations with LTDBCs and RTDBCs $\Delta S / \lambda$.

\section{Results and Discussion}

First, we choose $f_{i}=0.01$ and $d=20 \mathrm{~nm}$ to calculate the reflection spectrum $R$ and normalized GH shifts $S / \lambda$ with both LTDBCs and RTDBCs, respectively. The incident angle is considered as $\theta_{S P P s}$. The results are shown in Fig. 2. Furthermore, the difference of normalized GH shifts $\Delta S / \lambda$ is also plotted in Fig. 2.

Due to the interactions between SPPs and chiral TDBCs, normalized GH shifts $S / \lambda$ are amplified near the resonant frequencies of chiral TDBCs as shown in Fig. 2(a). They are 24 times of LTDBCs and 10 times of RTDBCs as the incident wavelength. Due to the difference resonant wavelength, the positions of the maximum $S$ for LTDBCs $(\lambda=577 \mathrm{~nm})$ and RTDBCs $(\lambda=582 \mathrm{~nm})$ do not overlap. As Fig. 2(b) shown, the difference of normalized GH shifts $\Delta S / \lambda$ can reach to 24 at $\lambda=577 \mathrm{~nm}$. It seems that the reflected beam with LTDBCs moves a lot, but the one with RTDBCs hardly moves at $\lambda=577 \mathrm{~nm}$. This phenomenon can be easily measured in experiment, and LTDBCs and RTDBCs are easily distinguished. Also, the working wavelength can be taken as $\lambda=582 \mathrm{~nm}$.

Then, we decrease the thickness $d$ of Au film to show how $\Delta S / \lambda$ changes. The thickness $d$ is changed from $20 \mathrm{~nm}$ to $50 \mathrm{~nm}$ with the step of $10 \mathrm{~nm}$. The results are shown in Fig. 3. For each thickness of the proposed system, $S_{i} / \lambda$ is also calculated and shown in the inserted picture. 

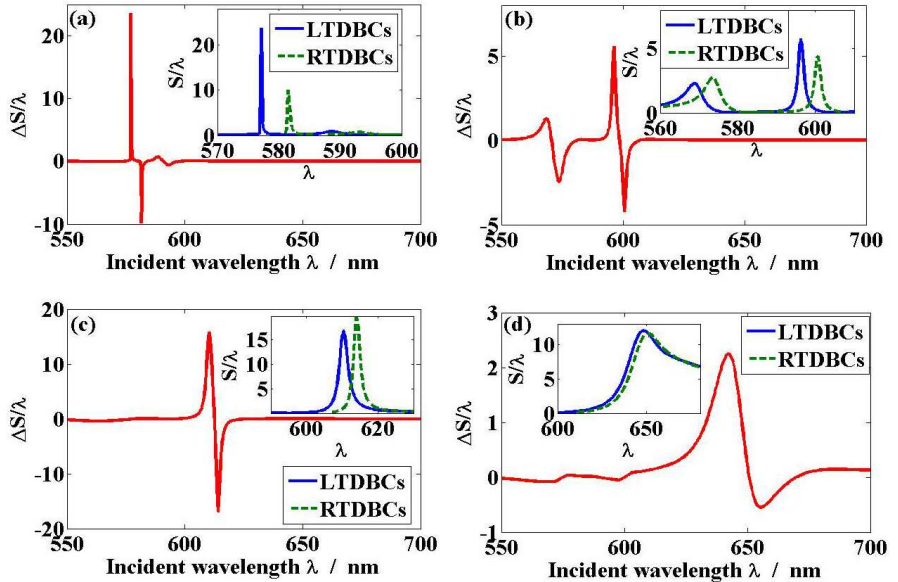

Fig. 3 (Color online). The difference of normalized GH shift $\Delta S / \lambda$ versus the incident wavelength $\lambda$ with $\mathrm{Au}$ film thickness $d$ changing from $20 \mathrm{~nm}$ to $50 \mathrm{~nm}$ with the step of $10 \mathrm{~nm}$, respectively. Normalized GH shifts $S_{i} / \lambda$ versus the incident wavelength $\lambda$ for each $d$ is in the inserted picture.
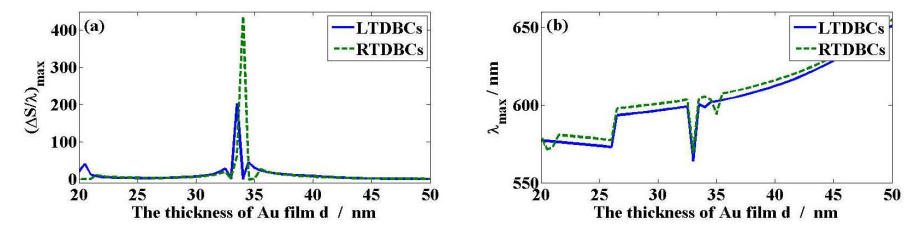

Fig. 4 (Color online). The maximum values (a) and the positions (b) of $\Delta S / \lambda$ for both LTDBCs and RTDBCs by changing $d$ with the step of $0.5 \mathrm{~nm}$, respectively.

As shown in Fig. 3, the working wavelength of our proposed structure is changed by the thickness of Au film $d$. At the same time, the value of $S_{i} / \lambda$ decreases. The dissipation of $\mathrm{Au}$ film increases sharply with the thickness of Au film increasing, while the decoupling conversion rate decreases. It means that there are more SPPs joining into the interaction between chiral TDBCs and $\mathrm{Au}$ film with the increase of $\mathrm{Au}$ film thickness. According to the analysis above, we can change the working wavelength by tuning the thickness of $\mathrm{Au}$ film. With the increase of $d$, the working wavelength has a red shift. Although there are more SPPs interact with chiral TDBCs, the intensity of $\Delta S / \lambda$ is reduced by the large dissipation. Comparing the intensity of $\Delta S / \lambda$, we also find that with the changes of $d$, there is an optimal thickness to make $\Delta S / \lambda$ largest. We pick the maximum values and the positions of $\Delta S / \lambda$ for both LTDBCs and RTDBCs by changing $d$ with the step of $0.5 \mathrm{~nm}$. The results are shown in Fig. 4.

As shown in Fig. 4(a), there is a little difference between the optimal thicknesses for LTDBCs and RTDBCs. The largest $\Delta S / \lambda$ is larger than 100 , which 

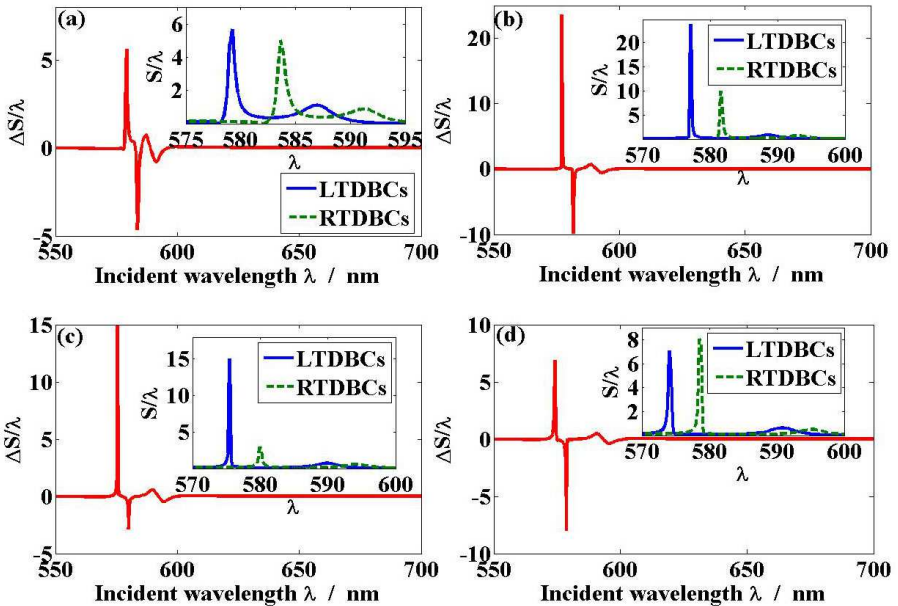

Fig. 5 (Color online). The difference of normalized GH shift $\Delta S / \lambda$ versus the incident wavelength $\lambda$ with coupling strength $f$ changing from 0.008 to 0.014 with the step of 0.002 , respectively. Normalized GH shifts $S_{i} / \lambda$ versus the incident wavelength $\lambda$ for each $f$ is in the inserted picture.

Table 1 The values of the peaks and dips in $\Delta S / \lambda$

\begin{tabular}{lllll}
\hline$f$ & $\mathbf{0 . 0 0 8}$ & $\mathbf{0 . 0 1}$ & $\mathbf{0 . 0 1 2}$ & $\mathbf{0 . 0 1 4}$ \\
\hline$\Delta S / \lambda(+)$ & $5.6(579 \mathrm{~nm})$ & $23.6(577 \mathrm{~nm})$ & $14.9(576 \mathrm{~nm})$ & $6.9(574 \mathrm{~nm})$ \\
$\Delta S / \lambda(-)$ & $4.7(584 \mathrm{~nm})$ & $9.8(582 \mathrm{~nm})$ & $2.9(580 \mathrm{~nm})$ & $8.0(579 n \mathrm{~nm})$ \\
\hline
\end{tabular}

can be significantly observed in the experiments. The position of the maximum of $\Delta S / \lambda$ for LTDBCs and RTDBCs almost do not overlap. The phenomena above are mainly caused by the different resonances for left TDBCs and right TDBCs. According to our calculations, we'd better to choose a suitable thickness of $\mathrm{Au}$ film to obtain obvious phenomenon for chiral TDBCs detecting in experiments.

Finally, we show the impact of the oscillator strength $f$ on $\Delta S / \lambda$. We change a small step of 0.002 to show the sensitivity of our proposed structure. $f$ is changed from 0.08 to 0.014 , and $\Delta S / \lambda$ versus $\lambda$ are shown in Fig. $5 . S_{i} / \lambda$ is also inserted in the figures for each $f$.

As Fig. 5 shown, the peaks and the dips of $\Delta S / \lambda$ have a slight shift (within $5 \mathrm{~nm})$ due to a quite small change of $f$. The values of the peaks and the dips in $\Delta S / \lambda$ changes a lot. This is because that $f$ has great influences on $\epsilon_{3}$ and $\theta_{S P P s}$. Hence, the value of $\Delta S / \lambda$ changes a lot. We list the values of the peaks and the dips in Tab. 1. We find that although $f$ changes a little 0.002 , the difference of GH shifts between peak values or dip values is no less than 5 times of the incident wavelength about $2.9 \mu \mathrm{m}$. This difference is easy to observe in experiment. 


\section{Summary}

In summary, we investigate a high sensitive chiral molecule detector based on GH shift in Kretschmann configuration involving chiral TDBCs. Fresnel equations and the stationary phase method are employed to calculate the lateral shift/GH shift. Due to the interaction between SPPs and the chiral TDBCs, the GH shifts in our proposed structures with two kind of chiral TDBCs are amplified at different wavelength. GH shift can reach to 24 times as the incident wavelength, and be easily observed in experiment. The working wavelength of the detector can be tuned by the thickness of $\mathrm{Au}$ film. We also find there is an optimal thickness to make $\Delta S$ largest, which is over 100 as the incident wavelength and can be easily observed in experiments. Furthermore, we discuss the oscillator strength $f$ and find that our proposed detector is quite sensitive with $f$. By changing 0.002 for $f$, the change of $\Delta S$ is no less than 5 times of the incident wavelength, which is about $2.9 \mu \mathrm{m}$ at our working wavelength. Our proposed structure is very sensitive and has potential applications in experiments.

\section{Ethical Approval}

This manuscript has not been submitted to anywhere.

\section{Consent to Participate}

All the authors agree to submit the manuscript to Nanoscale research Letters.

\section{Consent to Publish}

All the authors agree to publish the manuscript to Nanoscale research Letters.

\section{Authors Contributions}

Gang Song and Song Wang provide the idea. All the authors involve to prepare the manuscript. Song Wang and Gang Song have the same contributions to this work.

\section{Funding}

This research was supported by the Science and Technology Nova Plan of Beijing City (No. Z201100006820122), the Fundamental Research Funds for the Central Universities. 


\section{Competing Interests}

The authors declare no conflicts of interest

\section{Availability of data and materials}

All data generated or analysed during this study are included in this published article.

\section{References}

1. F. Goos and H. Hnchen. Ein neuer und fundamentaler versuch zur totalreflexion. $A n$ nalen Der Physik, 436(7-8):333-346, 1947.

2. Artmann K. Berechnung der seitenversetzung des totalreflektierten strahles. Annalen Der Physik, 2(1):87-102, 1948.

3. Knut Johansen, Ralph StaLberg, Ingemar LundstroM, and Bo Liedberg. Surface plasmon resonance: instrumental resolution using photo diode arrays. Measurement Science and Technology, 11(11):1630, 2000.

4. Chih Wei, Chen, Wen Chi, Lin, Lu Shing, Liao, Zheng Hung, Lin, Hai-Pang, and Chiang and. Optical temperature sensing based on the gooshanchen effect. Applied Optics, 46(22):5347-5351, 2007.

5. Wang Yi, Honggen Li, Zhuangqi Cao, Tianyi Yu, Qishun Shen, and He Ying. Oscillating wave sensor based on the goos-hanchen effect. Applied Physics Letters, 92(6):901, 2008.

6. Xiaobo Yin, Lambertus Hesselink, Zhaowei Liu, Nicholas Fang, and Zhang Xiang. Large positive and negative lateral optical beam displacements due to surface plasmon resonance. Applied Physics Letters, 85(3):372-374, 2004.

7. Haichun Zhou, Xi Chen, Peng Hou, and Chun Fang Li. Giant bistable lateral shift owing to surface-plasmon excitation in kretschmann configuration with a kerr nonlinear dielectric. Optics Letters, 33(11):1249-51, 2008.

8. Yuancheng Fan, Nian Hai Shen, Fuli Zhang, Zeyong Wei, Hongqiang Li, Qian Zhao, Quanhong Fu, Peng Zhang, Thomas Koschny, and Costas M. Soukoulis. Electrically tunable goos-h?nchen effect with graphene in the terahertz regime. Advanced Optical Materials, 4(11):1824-1828, 2016.

9. Ji Hun Kang, Sheng Wang, Zhiwen Shi, Wenyu Zhao, Eli Yablonovitch, and Feng Wang. Goos-hanchen shift and even-odd peak oscillations in edge-reflections of surface polaritons in atomically thin crystals. Nano Letters, 17(3):1768-1774, 2017.

10. M. Alamri, M. Suhail Zubairy, and Xiaodong Zeng. Tunable goos-hanchen shift from graphene ribbon array. Optics Express, 25(20):23579-23588, 2017.

11. Joong Sung Lee, Trung Huynh, Su Yong Lee, Kwang Geol Lee, Jinhyoung Lee, Mark Tame, Carsten Rockstuhl, and Changhyoup Lee. Quantum noise reduction in intensitysensitive surface-plasmon-resonance sensors. Phys.rev.a, 96(3):033833, 2017.

12. Kunwei Pang, Haihong Li, Gang Song, and Pengfei Zhang. Splitting in lateral shift induced by strong coupling in kretschmann configuration involving molecular j-aggregates. Modern Physics Letters B, 33(6):1950370, 2019.

13. J. Bellessa, C. Bonnand, J. C. Plenet, and J. Mugnier. Strong coupling between surface plasmons and excitons in an organic semiconductor. Physical Review Letters, 93(3):036404, 2004.

14. Qingling Ouyang, Shuwen Zeng, Li Jiang, Liying Hong, Gaixia Xu, Xuan-Quyen Dinh, Jun Qian, Sailing He, Junle Qu, and Philippe Coquet. Sensitivity enhancement of transition metal dichalcogenides/silicon nanostructure-based surface plasmon resonance biosensor. Scientific Reports, 6:28190, 2016.

15. Julien Moreau Jean-Francois Bryche Aurore Olivero? Gregory Barbillon Anne-Lise Coutrot Bernard Bartenlian Mitradeep Sarkar, Mondher Besbes and Michael Canva. Hybrid plasmonic mode by resonant coupling of localized plasmons to propagating plasmons in a kretschmann configuration. ACS Photonics, 2:237-245, 2015. 
16. H. Memmi, O. Benson, S. Sadofev, and S. Kalusniak. Strong coupling between surface plasmon polaritons and molecular vibrations. Physical Review Letters, 118(12):126802, 2017.

17. Mario Hentschel, Martin Schäferling, Xiaoyang Duan, Harald Giessen, and Na Liu. Chiral plasmonics. Science advances, 3(5):e1602735, 2017.

18. David Patterson, Melanie Schnell, and John M Doyle. Enantiomer-specific detection of chiral molecules via microwave spectroscopy. Nature, 497(7450):475, 2013.

19. Jino George and K George Thomas. Surface plasmon coupled circular dichroism of au nanoparticles on peptide nanotubes. Journal of the American Chemical Society, 132(8):2502-2503, 2010.

20. Mengtao Sun, Zhenglong Zhang, Peijie Wang, Qiang Li, Fengcai Ma, and Hongxing $\mathrm{Xu}$. Remotely excited raman optical activity using chiral plasmon propagation in ag nanowires. Light: Science \& Applications, 2(11):e112, 2013.

21. Anton Kuzyk, Robert Schreiber, Zhiyuan Fan, Günther Pardatscher, Eva-Maria Roller, Alexander Högele, Friedrich C Simmel, Alexander O Govorov, and Tim Liedl. Dnabased self-assembly of chiral plasmonic nanostructures with tailored optical response. Nature, 483(7389):311, 2012.

22. Ben M Maoz, Yulia Chaikin, Alexander B Tesler, Omri Bar Elli, Zhiyuan Fan, Alexander O Govorov, and Gil Markovich. Amplification of chiroptical activity of chiral biomolecules by surface plasmons. Nano letters, 13(3):1203-1209, 2013.

23. Shunping Zhang, Hong Wei, Kui Bao, Ulf Håkanson, Naomi J Halas, Peter Nordlander, and Hongxing Xu. Chiral surface plasmon polaritons on metallic nanowires. Physical review letters, 107(9):096801, 2011.

24. Alexander O Govorov, Zhiyuan Fan, Pedro Hernandez, Joseph M Slocik, and Rajesh R Naik. Theory of circular dichroism of nanomaterials comprising chiral molecules and nanocrystals: plasmon enhancement, dipole interactions, and dielectric effects. Nano letters, 10(4):1374-1382, 2010.

25. Thibault Chervy, Stefano Azzini, Etienne Lorchat, Shaojun Wang, Yuri Gorodetski, James A Hutchison, Ste?phane Berciaud, Thomas W Ebbesen, and Cyriaque Genet. Room temperature chiral coupling of valley excitons with spin-momentum locked surface plasmons. ACS Photonics, 5(4):1281-1287, 2018.

26. MH Alizadeh and Bjo?rn M Reinhard. Transverse chiral optical forces by chiral surface plasmon polaritons. ACS Photonics, 2(12):1780-1788, 2015.

27. Quanbo Jiang, Aline Pham, Martin Berthel, Serge Huant, Joel Bellessa, Cyriaque Genet, and Aurélien Drezet. Directional and singular surface plasmon generation in chiral and achiral nanostructures demonstrated by leakage radiation microscopy. ACS Photonics, 3(6):1116-1124, 2016.

28. Xiang Lan and Qiangbin Wang. Self-assembly of chiral plasmonic nanostructures. Advanced Materials, 28(47):10499-10507, 2016.

29. Gang Song, Jiaqi Guo, Gaoyan Duan, Rongzhen Jiao, and Li Yu. Interactions between a single metallic nanoparticle and chiral molecular j-aggregates in the strong coupling regime and the weak coupling regime. Nanotechnology, 31(34):345202, jun 2020.

30. Gang Song Tiantian He, Qihui Ye. Enhanced circular dichroism of tdbc in a metallic hole array structure. Chinese Physics B, 29(9):97306, 2020.

31. Fan Ye, Juan M. Merlo, Michael J. Burns, and Michael J. Naughton. Optical and electrical mappings of surface plasmon cavity modes. Nanophotonics, 3(1-2):33-49, 2014.

32. Yachao Liu, Yougang Ke, Hailu Luo, and Shuangchun Wen. Photonic spin hall effect in metasurfaces: a brief review. Nanophotonics, 6(1), 2016.

33. Farzaneh Afshinmanesh, Justin S. White, Wenshan Cai, and Mark L. Brongersma. Measurement of the polarization state of light using an integrated plasmonic polarimeter. Nanophotonics, 1(2):125-129, 2012.

34. Ahmad Darweesh, Stephen Bauman, Desalegn Debu, and Joseph Herzog. The role of rayleigh-wood anomalies and surface plasmons in optical enhancement for nanogratings. Nanomaterials, 8(10), 2018.

35. Yindi Wang, Hongxia Liu, Shulong Wang, Ming Cai, Haifeng Zhang, and Yanbin Qiao. Electrical phase control based on graphene surface plasmon polaritons in mid-infrared. Nanomaterials, 10(3), 2020. 
36. Andrey A. Vyshnevyy and Dmitry Yu. Fedyanin. Hybrid electro-optical pumping of active plasmonic nanostructures. Nanomaterials, 10(5):856, 2020.

37. Yuxia Tang, Yingzhou Huang, Linhong Qv, and Yurui Fang. Electromagnetic energy redistribution in coupled chiral particle chain-film system. Nanoscale Research Letters, 13(1):194, 2018.

38. Giant tunable circular dichroism of large-area extrinsic chiral metal nanocrescent arrays. Nanoscale Research Letters, 14(1):1-7, 2019.

39. Niculina Peica, Christian Thomsen, and Janina Maultzsch. Studying the local character of raman features of single-walled carbon nanotubes along a bundle using ters. Nanoscale Research Letters, 6(1):174-174, 2011.

40. Edward D Palik. Handbook of optical constants of solids, volume 3. Academic press, 1998.

41. Stefan Alexander Maier. Plasmonics: Fundamentals and Applications, volume 1. Springer press, 2007. 


\section{Figures}

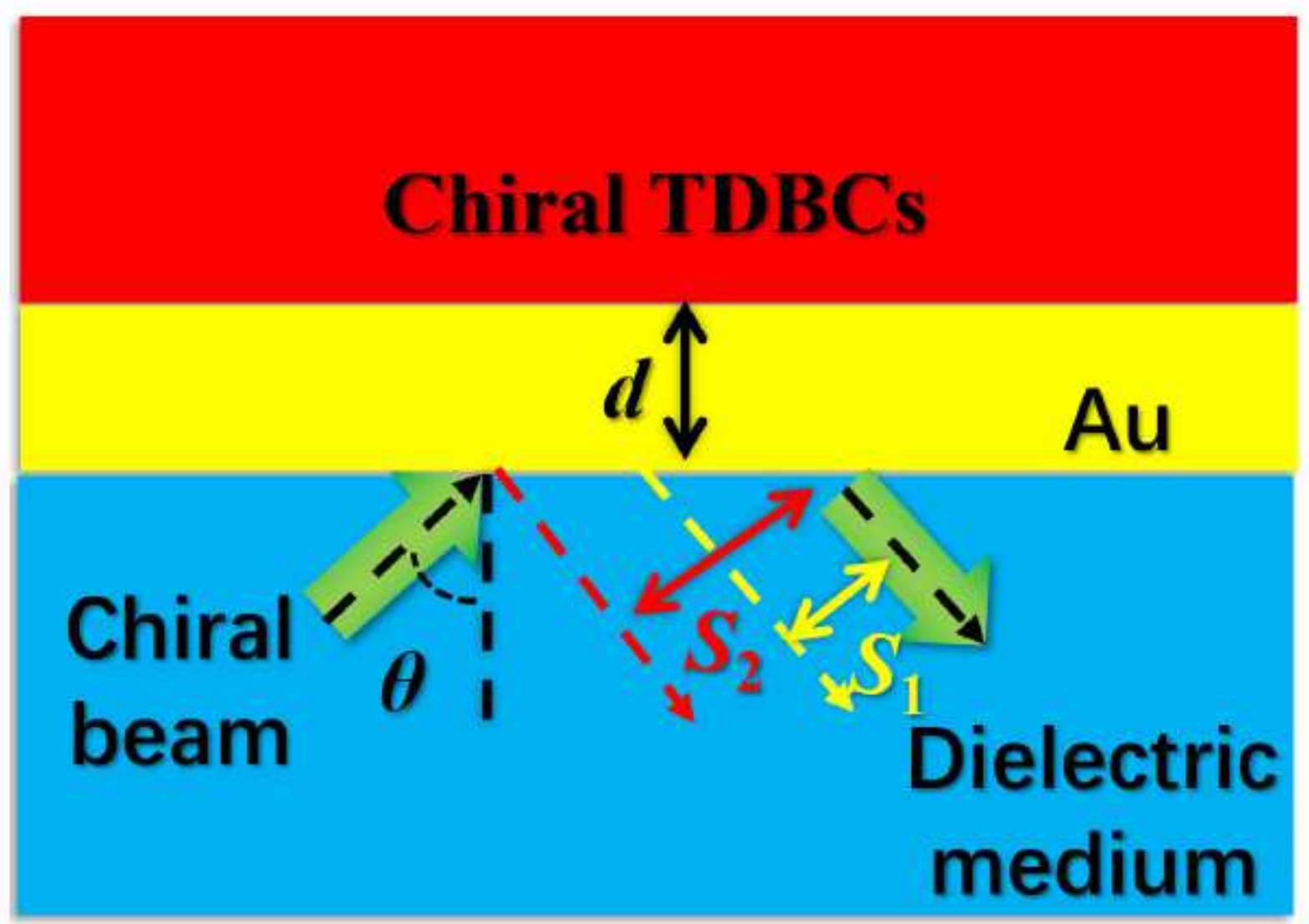

Figure 1

(Color online). The sketch of our calculation model. 


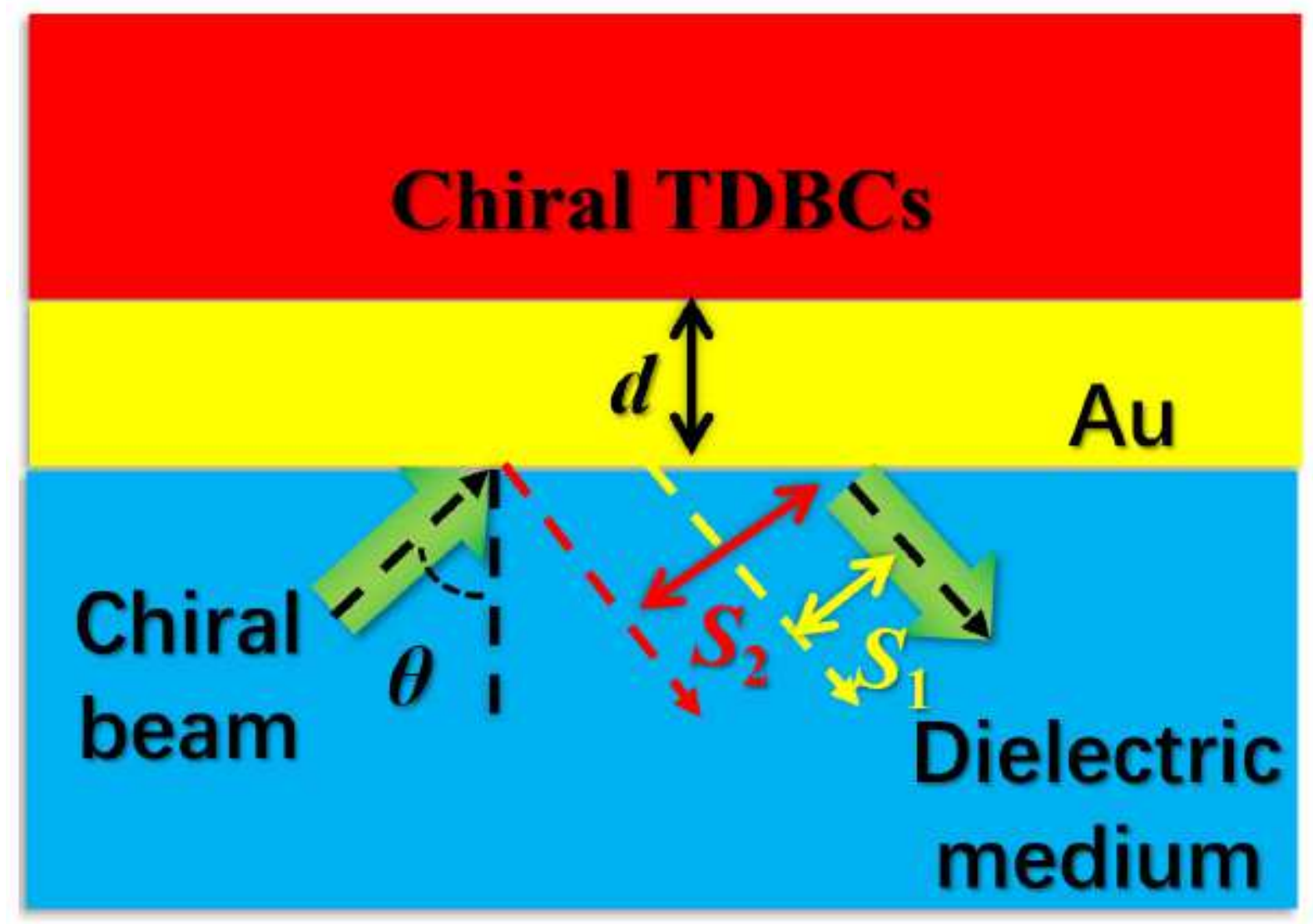

Figure 1

(Color online). The sketch of our calculation model.
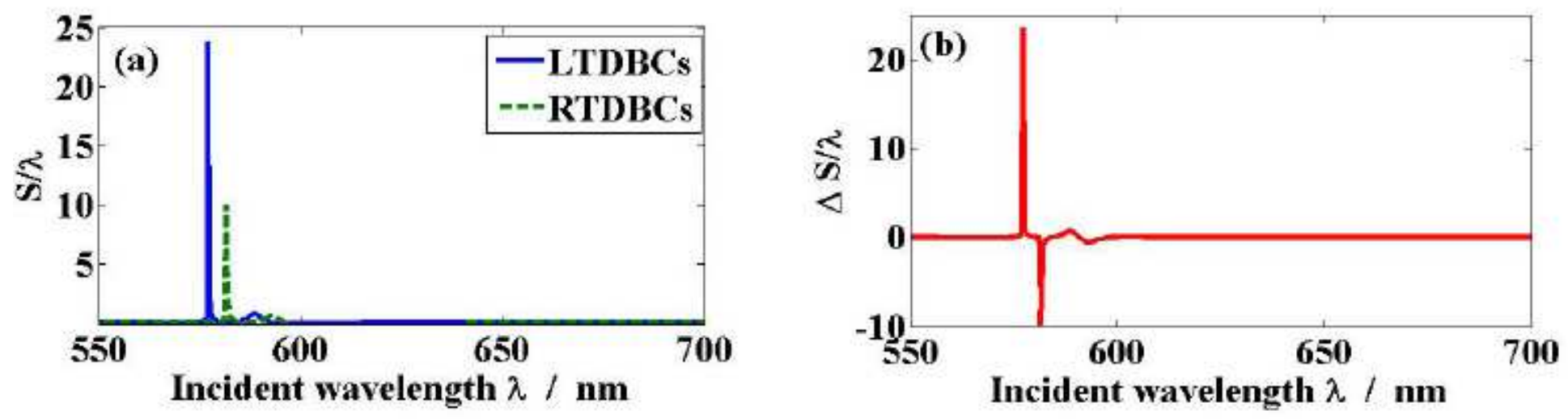

Figure 2

Normalized GH shifts $\mathrm{Si} / \lambda$ (a) and the difference of normalized GH shift $\Delta S / \lambda$ (b) versus the incident wavelength $\lambda$, respectively. 

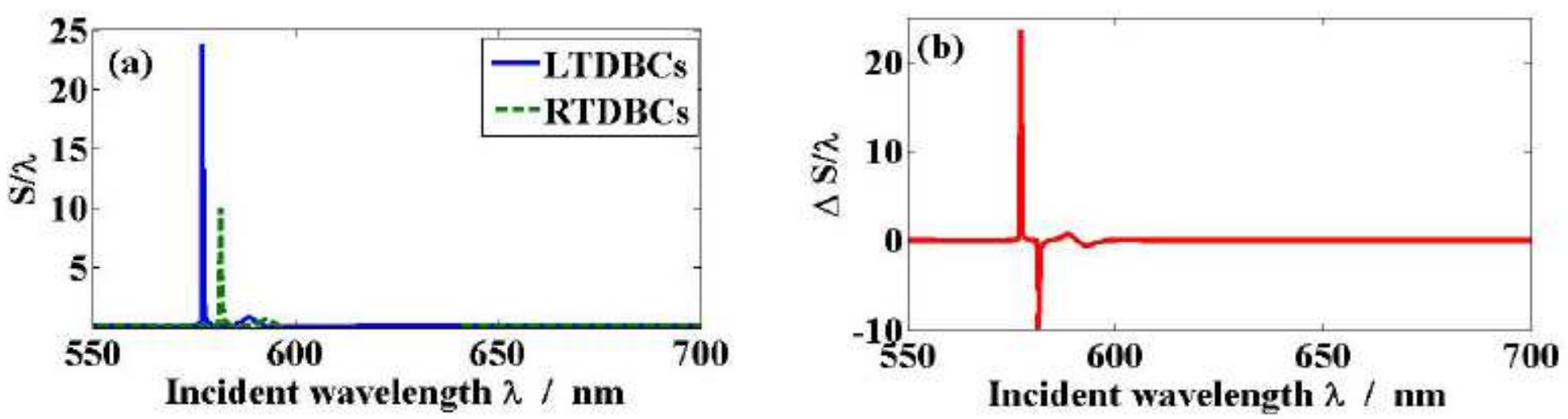

Figure 2

Normalized GH shifts $\mathrm{Si} / \lambda(\mathrm{a})$ and the difference of normalized GH shift $\Delta S / \lambda$ (b) versus the incident wavelength $\lambda$, respectively.
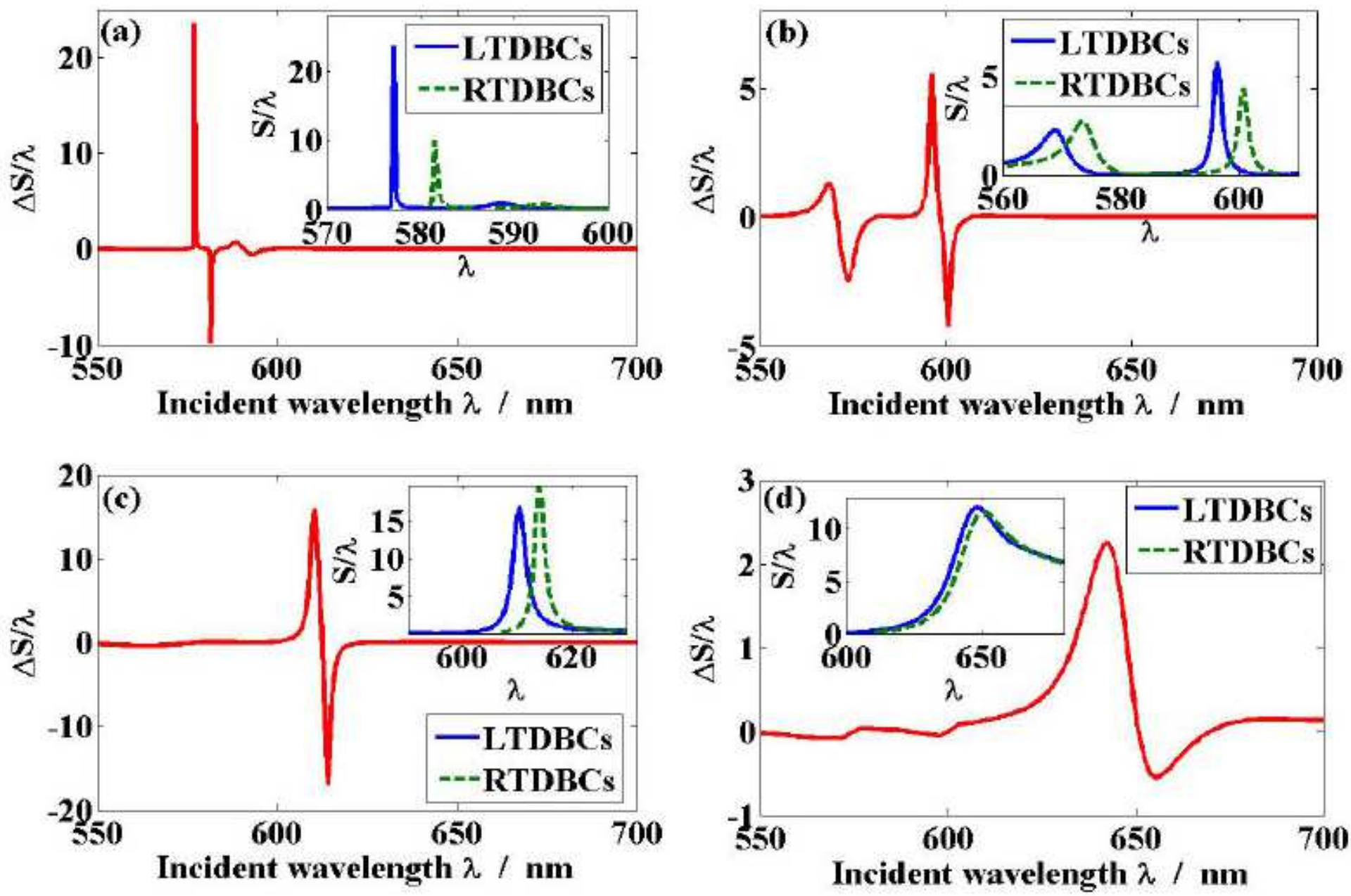

Figure 3

(Color online). The difference of normalized GH shift $\Delta S / \lambda$ versus the incident wavelength $\lambda$ with Au film thickness $d$ changing from $20 \mathrm{~nm}$ to $50 \mathrm{~nm}$ with the step of $10 \mathrm{~nm}$, respectively. Normalized $\mathrm{GH}$ shifts $\mathrm{Si} / \lambda$ versus the incident wavelength $\lambda$ for each $d$ is in the inserted picture. 

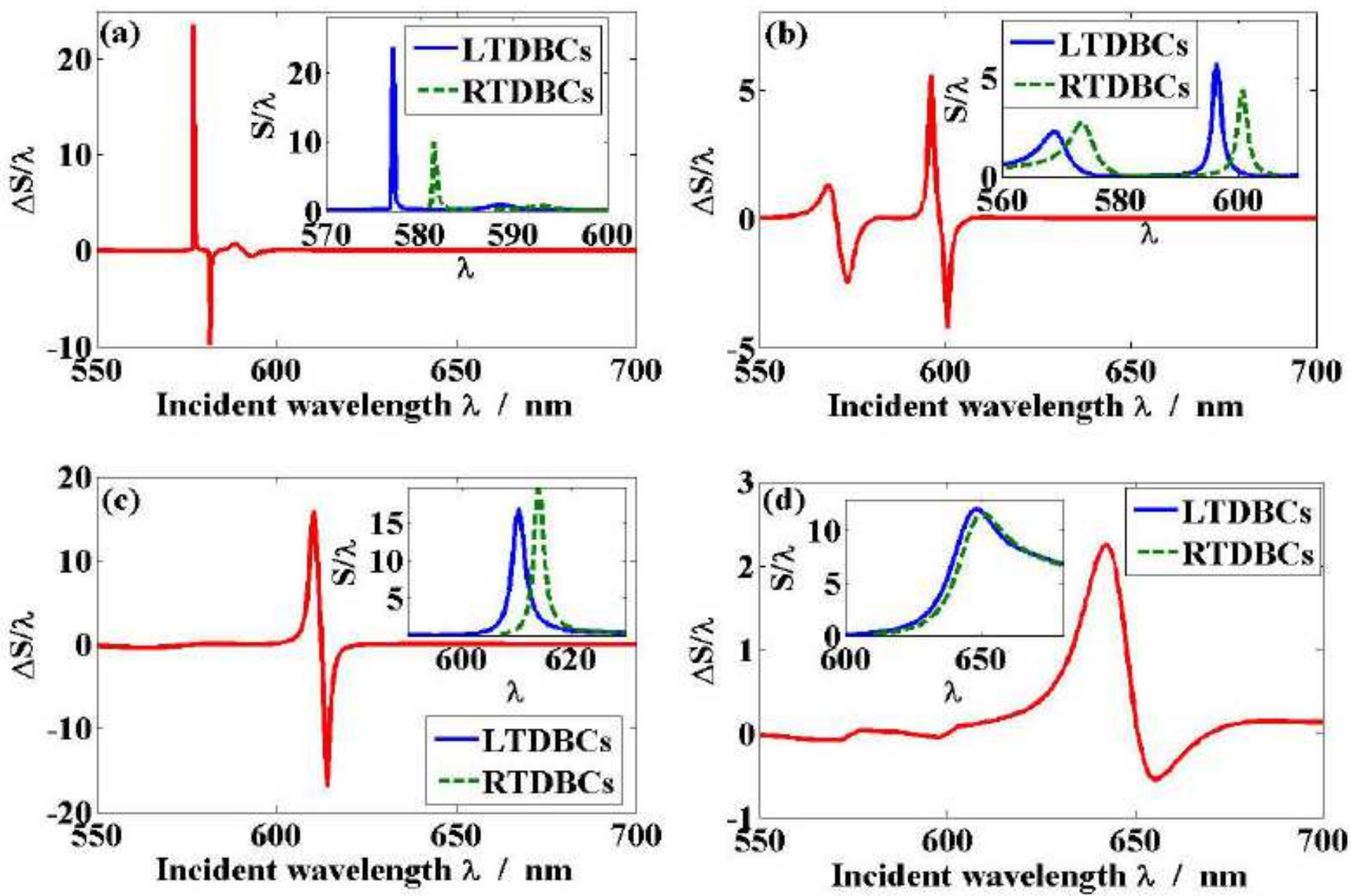

Figure 3

(Color online). The difference of normalized GH shift $\Delta S / \lambda$ versus the incident wavelength $\lambda$ with Au film thickness $d$ changing from $20 \mathrm{~nm}$ to $50 \mathrm{~nm}$ with the step of $10 \mathrm{~nm}$, respectively. Normalized $\mathrm{GH}$ shifts $\mathrm{Si} / \lambda$ versus the incident wavelength $\lambda$ for each $d$ is in the inserted picture.
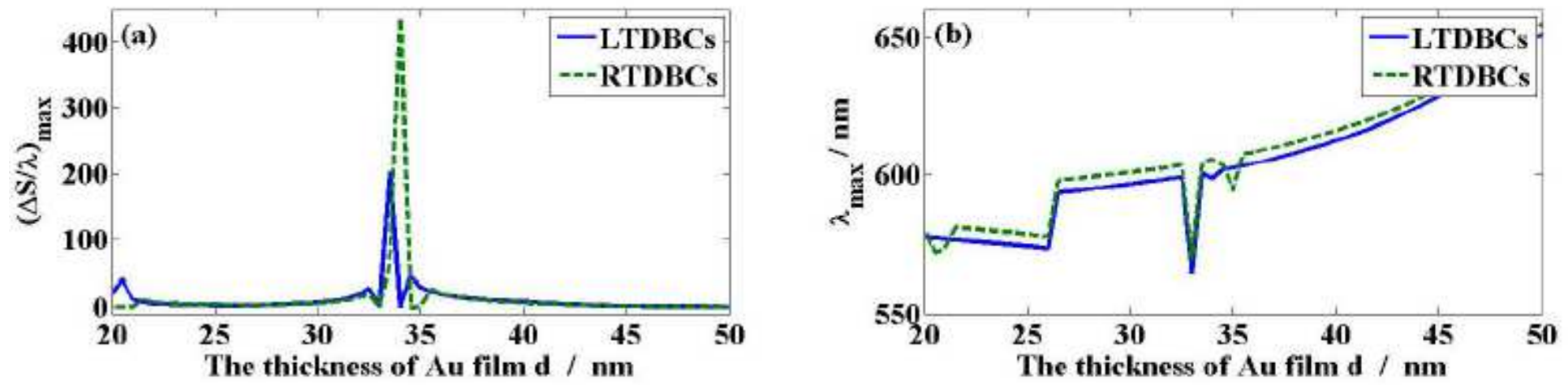

Figure 4

(Color online). The maximum values (a) and the positions (b) of $\Delta S / \lambda$ for both LTDBCs and RTDBCs by changing $d$ with the step of $0.5 \mathrm{~nm}$, respectively. 

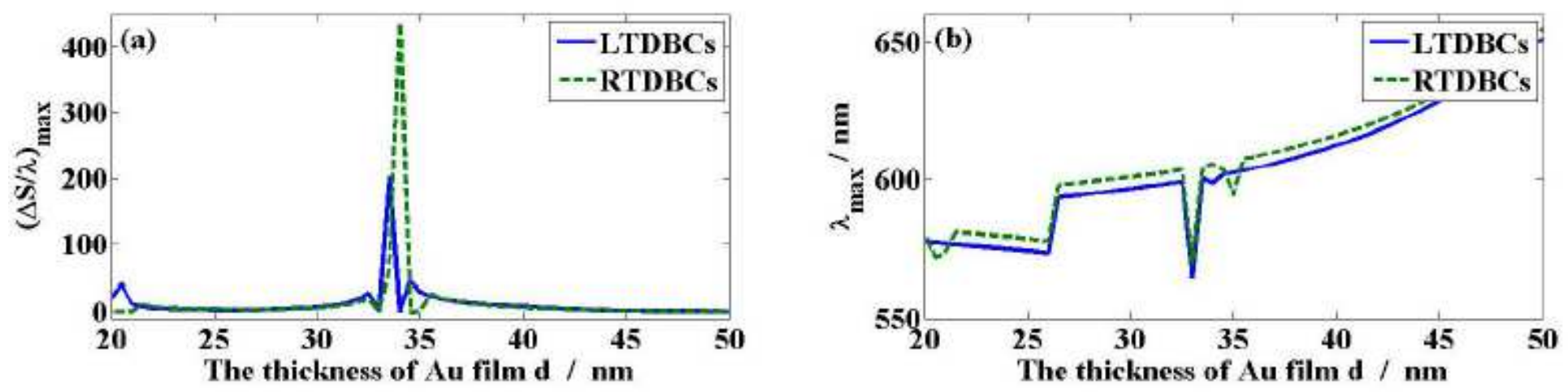

Figure 4

(Color online). The maximum values (a) and the positions (b) of $\Delta S / \lambda$ for both LTDBCs and RTDBCs by changing $\mathrm{d}$ with the step of $0.5 \mathrm{~nm}$, respectively.

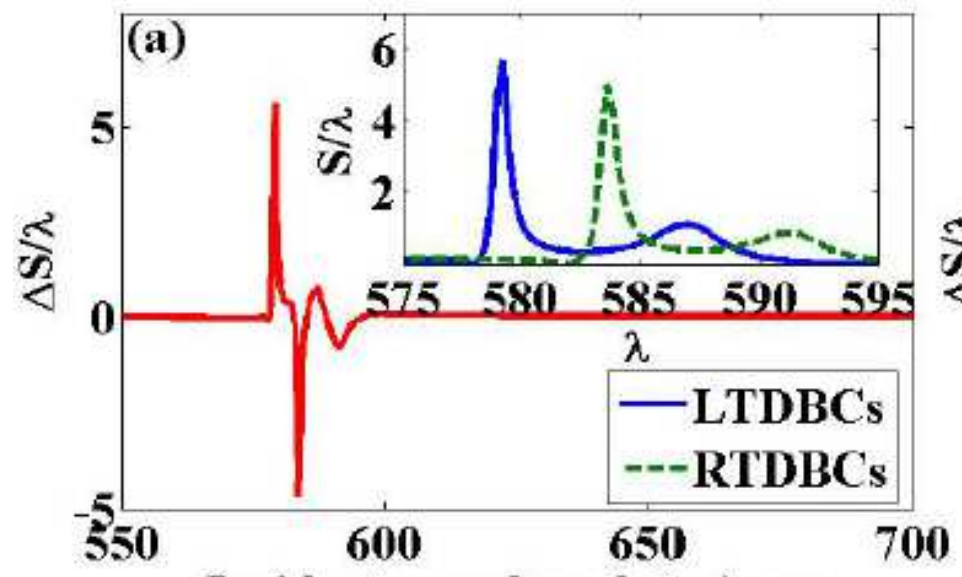

Incident wavelength $\lambda / \mathrm{nm}$

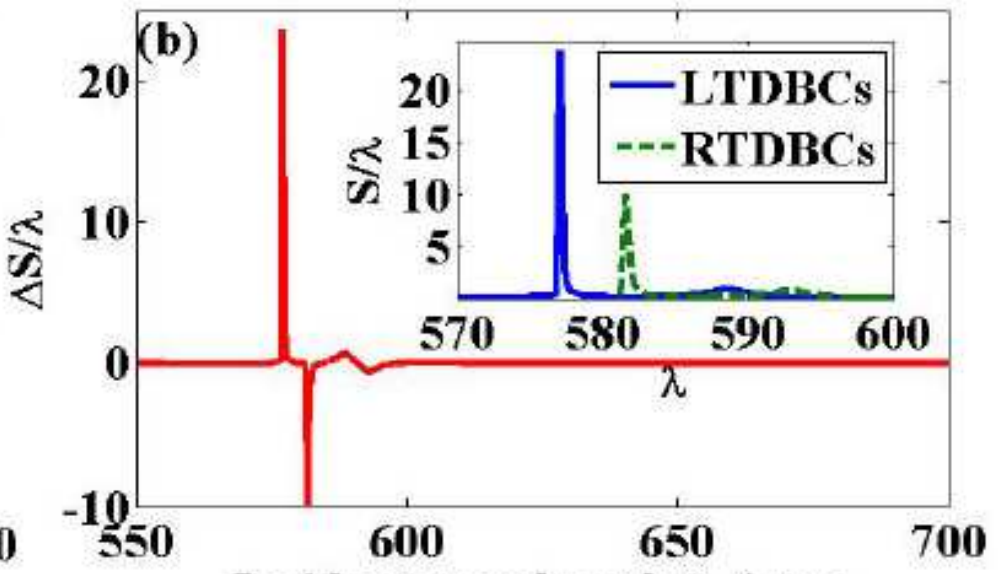

Incident wavelength $\lambda / \mathbf{~ n m}$
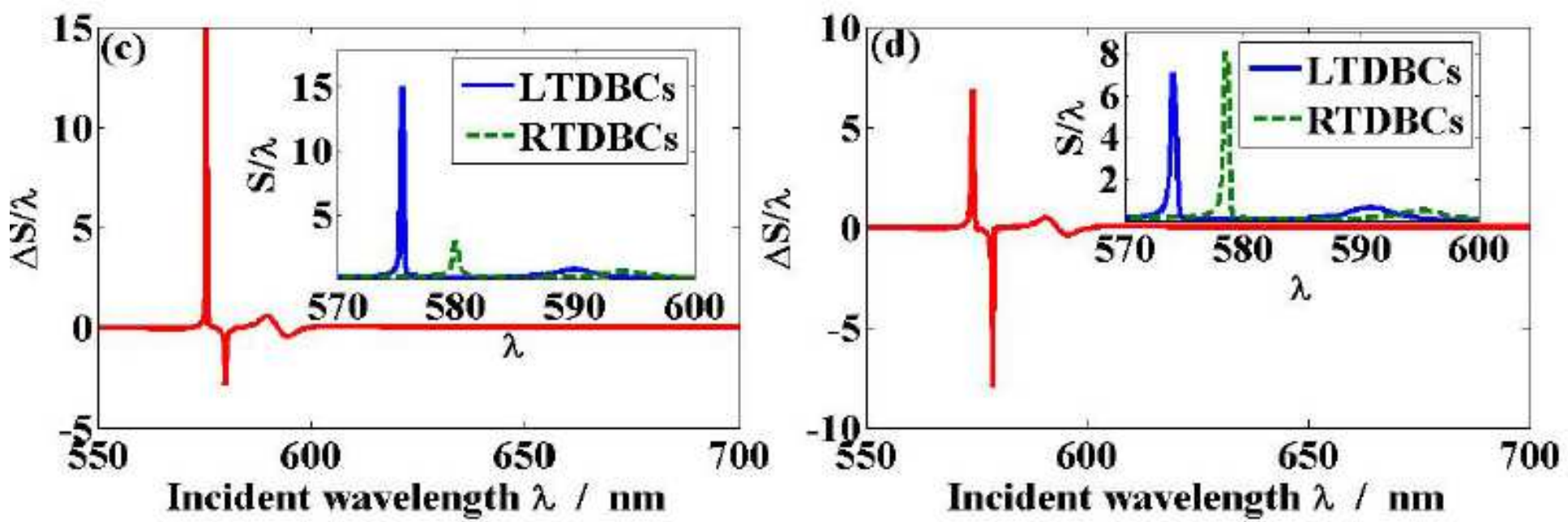

Figure 5

(Color online). The difference of normalized GH shift $\Delta S / \lambda$ versus the incident wavelength $\lambda$ with coupling strength $f$ changing from 0.008 to 0.014 with the step of 0.002 , respectively. Normalized $\mathrm{GH}$ shifts $\mathrm{Si} / \lambda$ versus the incident wavelength $\lambda$ for each $f$ is in the inserted picture. 


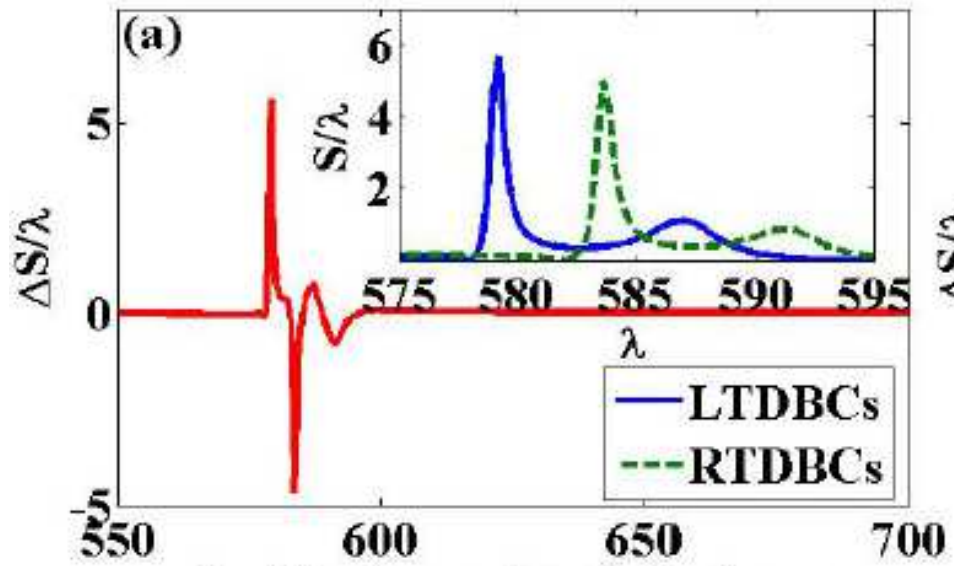

Incident wavelength $\lambda / \mathrm{nm}$

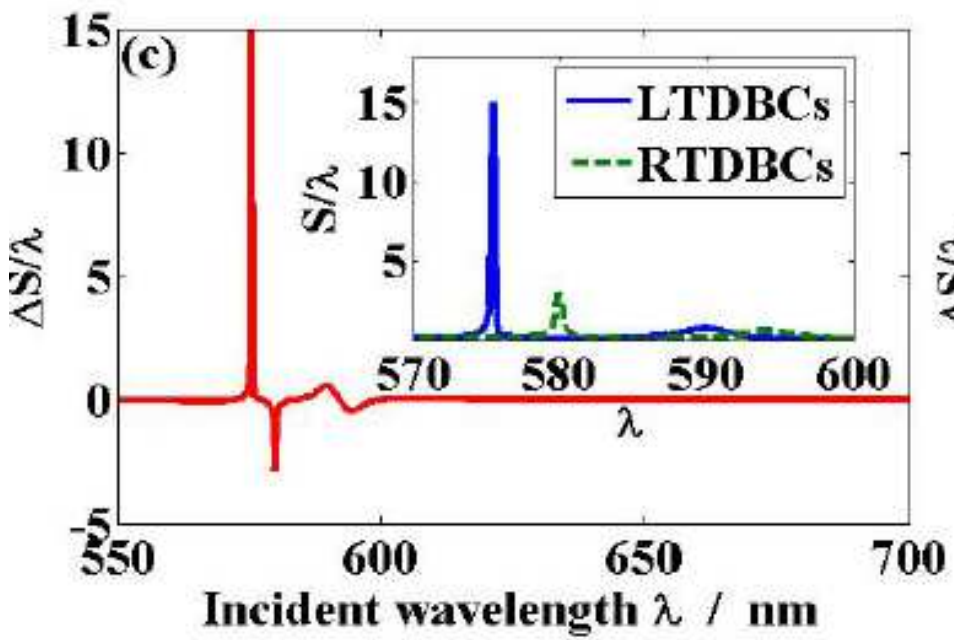

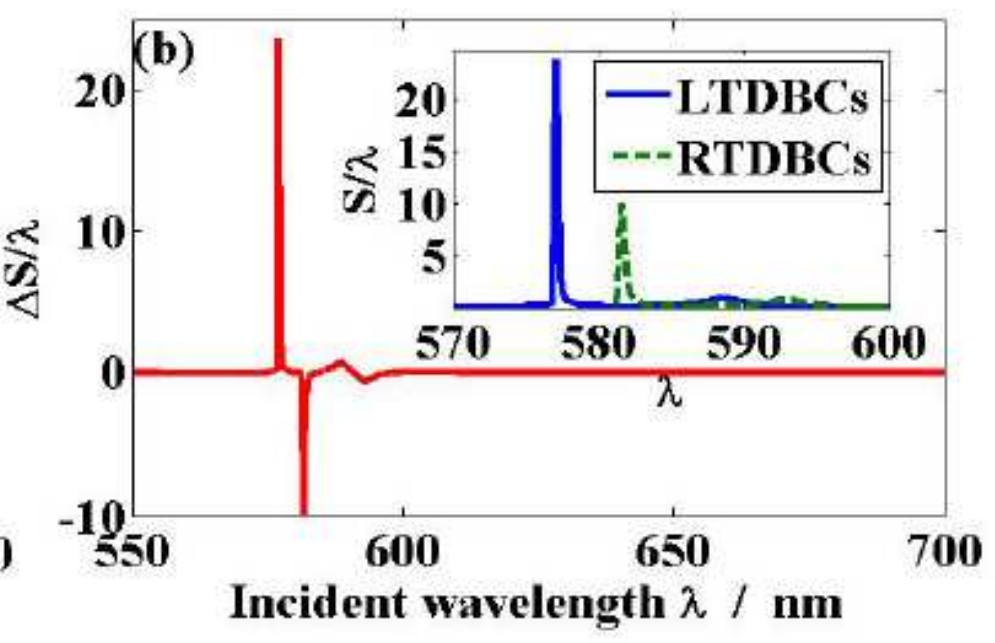

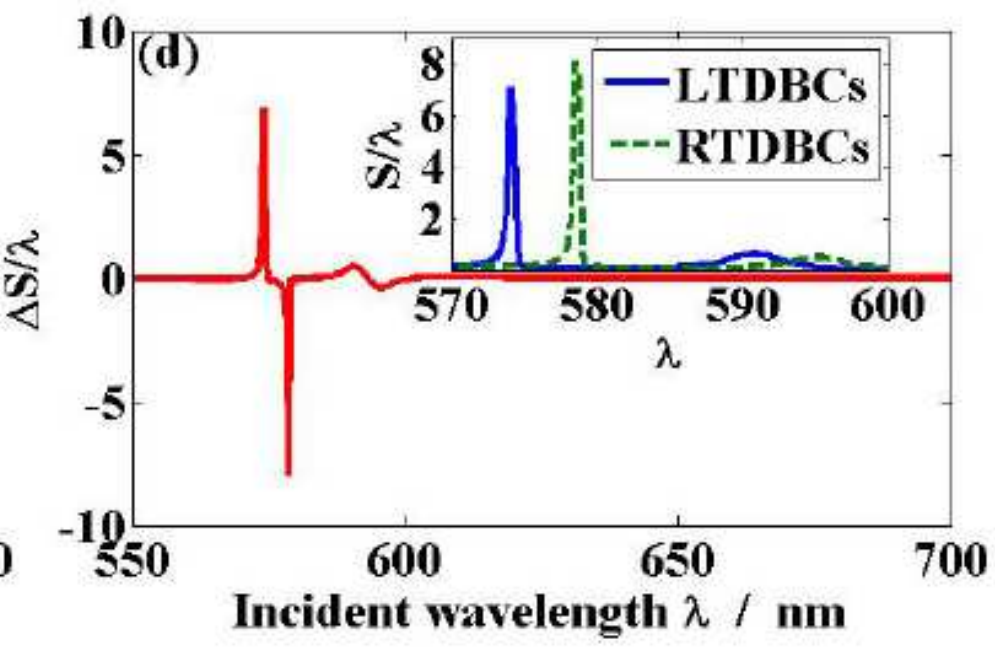

Figure 5

(Color online). The difference of normalized GH shift $\Delta S / \lambda$ versus the incident wavelength $\lambda$ with coupling strength $f$ changing from 0.008 to 0.014 with the step of 0.002 , respectively. Normalized GH shifts $\mathrm{Si} / \lambda$ versus the incident wavelength $\lambda$ for each $f$ is in the inserted picture. 